\title{
Analysis of spectroscopic diffuse reflectance plots for different skin conditions
}

\author{
Shanthi Prince* and S. Malarvizhi \\ Department of Electronics and Communication Engineering, SRM University, Kattankulathur, India
}

\begin{abstract}
Optical means of characterizing tissues have gained importance due to its noninvasive nature. Spectral characteristics of the components provide useful information to identify the components, because different chromophores have different spectroscopic responses to electromagnetic waves of a certain energy band. The purpose of this study is to determine whether visible/near-infrared diffuse reflectance spectroscopy can be used to non-invasively characterize skin diseases in vivo.

An optical fiber spectrometer is set up for obtaining diffuse reflectance spectra. The method involves exposure of skin surface to white light produced by an incandescent source. The back scattered photons emerging from various layers of tissue are detected by spectrometer resulting in diffuse reflectance spectra.

For the present study different skin conditions like - warts, vitiligo, thrombus (due to injury) and angioma are chosen. The spectral data obtained from the scan are plotted and compared. More or less, the shapes of the spectral curves for various skin conditions resemble. In order to characterize and differentiate different diseased state spectral analysis based on Ratio analysis, Student's $t$-tests and difference plot are carried out.

Based on the analysis the relative spectral intensity changes are quantified and the spectral shape changes are enhanced and more easily visualized on the spectral curves, thus assisting in differentiating the normal tissue from the one affected by disease.

Keywords: Diffuse reflectance, spectroscopy, control skin, ratio-analysis, spectral analysis
\end{abstract}

\section{Introduction}

Advances in the understanding of light transport through turbid media during the 1990s led to the development of technologies based on diffuse optical spectroscopy and diffuse optical imaging $[1,2]$. There has recently been significant interest in developing optical spectroscopy as a tool to augment the current protocols for cancer diagnosis [3], as it has the capability to probe changes in the biochemical composition of tissue that accompany disease progression. Many robust and powerful combined optical diagnostic techniques, such as fluorescence or light scattering have been recently designed [4]. Raman spectroscopy is a great tool for studying the structure and dynamic function of biologically important molecules [5].

A variety of materials in skin, absorbs mid-IR light $(>2500 \mathrm{~nm})$, thus providing an insight into skin biochemistry. But, if the sample thickness is greater than $10-15 \mu \mathrm{m}$, mid-IR light is completely absorbed. Therefore, the diagnostic potential of mid-IR spectroscopy in vivo is limited, In contrast, near-IR light is scattered to a much greater extent than it is absorbed.

The near-IR region is often sub-divided into the short $(680-1100 \mathrm{~nm})$ and long $(1100-2500 \mathrm{~nm})$ nearIR wavelengths, based upon the technology required to analyze light in these wavelength regions. At

\footnotetext{
${ }^{*}$ Corresponding author: Shanthi Prince, Department of Electronics and Communication Engineering, SRM University, Kattankulathur, India. Tel.: +91 9444962179; Fax: +91 44 27452343, E-mail: shanthiprince@ktr.srmuniv.ac.in.
} 
shorter near-IR wavelengths, oxy- and deoxyhemoglobin, myoglobin and cytochromes dominate the spectra, and their absorptions are indicative of regional blood flow and oxygen consumption [6].

Diffuse reflectance spectroscopy studies the changes in the optical properties associated with disease or therapy. Reflectance measurements can be performed with very short light pulses (time-domain), an intensity modulated source (frequency-domain), or a steady state broadband or monochromatic source. In this study we have used visible/near infrared steady-state in vivo diffuse reflectance measurements to characterize tissue and provide clinically useful diagnostic information. A number of clinical studies performed with tissues such as colon [7], bladder [8], breast [9] and skin [10] demonstrate that diffuse reflectance spectra contain diagnostically useful information.

Near-IR light is scattered to a much greater extent than it is absorbed, making tissues relatively transparent to near-IR light, thus allowing the examination of much larger volumes of tissue [11] and the potential for in-vivo studies.

Mapping oxygen saturation of blood in skin will give useful information for skin disorder diagnosis [12]. The oxy-hemoglobin, deoxy-hemoglobin and oxygen saturation in palm under normal and venous occlusions are estimated and mapped from multi-spectral reflectance data [13]. Previously, we have presented preliminary studies that assess the diagnostic power of diffuse reflectance spectroscopy [14].

Many benign skin diseases resemble malignancies upon visual examination. As a consequence, histopathological analysis of skin biopsies remains the standard for confirmation of a diagnosis. Visible/near-infrared (NIR) spectroscopy may be that tool which could be utilized for characterization of skin diseases prior to biopsy.

A non-invasive tool for skin disease diagnosis would be a useful clinical adjunct. The purpose of this study is to determine whether the information obtained from visible/near-IR spectroscopy for a variety of skin diseases will enable us to characterize the tissue be used to non-invasively characterize skin diseases.

\section{Materials and methods}

\subsection{Diffuse reflectance spectroscopy}

Light reflected from a surface consists of specularly reflected and diffusely reflected components. The intensity of the specular component is largely determined by the surface properties of the sample and is generally not of analytical utility. The intensity of the diffuse component, which includes the contributions from the absorbance of light by the specimen and the scattering of light by the specimen and the substrate, can be used to determine the concentration of the indicator species [6].

Human skin is an inhomogeneous absorbing medium with strong scattering properties. Light interaction with such a medium has a complicated character [15]. The horny skin layer (stratum corneum) has an average refractive index higher than that of air. This layer is responsible for the specular reflection of light on the skin/air interface (5-7\% of the total incident light). A significant part (93-95\% of the light is scattered and absorbed by the remaining layers of the skin, i.e., the epidermis, dermis, basal lamina, blood vessels, etc. [15-17].

A diffuse reflectance spectrum arises from light that has been scattered multiple times within the sample of interest. The spectral features of light diffusely reflected from tissue depend on its scattering and absorption properties. Diffuse reflectance spectroscopy studies the changes in these optical properties associated with disease or therapy. The spectrum qualitatively identifies and quantitatively determines 
concentrations of various chromophores in the tissue under study [18]. Any subtle changes in these will reflect on the spectrum. Thus optical spectrum from the tissue yield diagnostic information based on the biochemical composition and structure of the tissue. Different skin and sub-surface tissues have distinct or unique reflectance pattern which help us differentiate normal and diseased tissues [19]. In the visible spectral range $(400-700 \mathrm{~nm})$, penetration depth is between $0.5-2.5 \mathrm{~mm}$ [20]. In this case, both absorption and scattering play a dominant role in the formation of the diffuse reflectance spectrum. In the spectral range 500-600 nm, absorption is even lower, scattering dominates absorption, and the penetration depth can be up to $8-10 \mathrm{~mm}$ [16]. Light within the skin is entirely diffuse, thus the diffuse reflectance increases. In the near-IR spectral range, skin reflectance increases up to 800-900 nm and then decreases due to increasing water absorption $[17,20]$.

In diffuse reflectance spectroscopy, the experimental setup used influence the intensity and spectral features of the diffuse reflectance spectra. Also, measurements depend on the geometry of the optical fibers used.

\subsection{Instrumentation}

The detection and analysis of the optical signal requires instrumentation. Basically, a spectrophotometric measurement is to be done so that the obtained spectra can be related to the molecular composition and structure of biochemical species in the sample of interest. The schematic diagram of the visible/near-infrared (NIR) spectroscopy system is shown in Fig. 1. It consists of tungsten halogen light source (LS-1, Ocean Optics, Inc.) [21] which is a versatile white-light source optimized for the VISNIR (360-2500 nm) wavelength range, fiber optic reflectance probe (R400, Ocean Optics, Inc.) [21], spectrometer (USB4000, Ocean Optics, Inc.) [21] with CCD device, and a computer with an acquisition software (Spectra Suite, Ocean Optics, Inc.) [21]. The USB4000 is responsive from 200 to $1100 \mathrm{~nm}$,

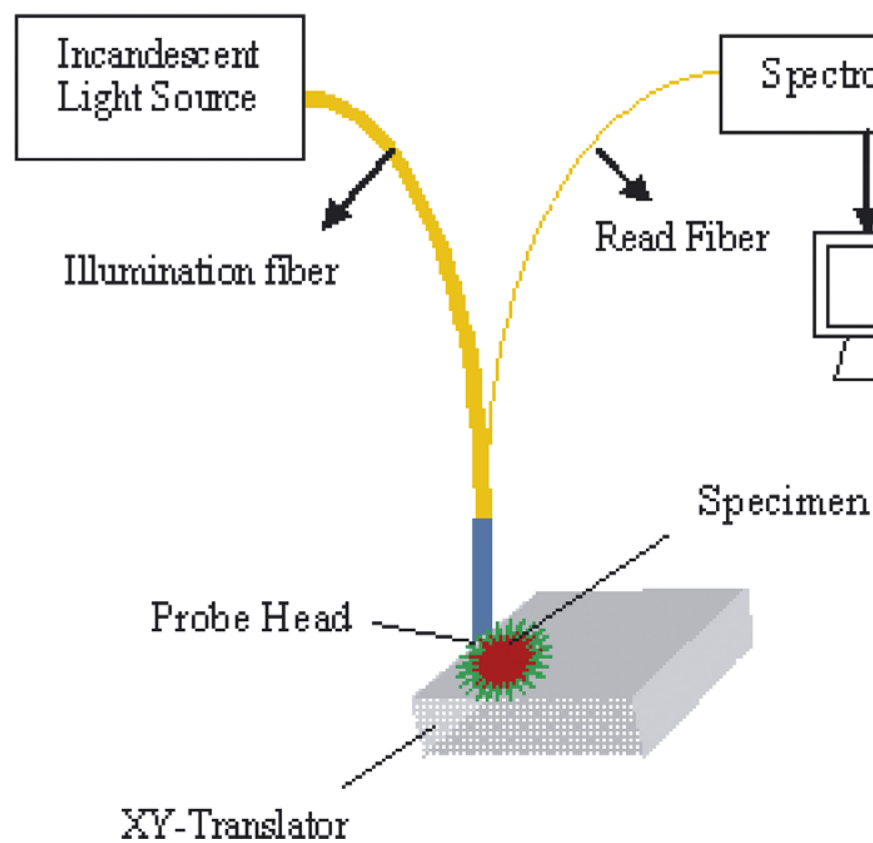

Fig. 1. The schematic diagram of Visible/near-infrared (NIR) spectroscopy system based on diffused reflectance. 
with an optical resolution of $\sim 0.3 \mathrm{~nm}$ (FWHM). It consists of 3648 element detector with shutter, high speed electronics and interface capabilities. The reflectance probe (R400) consists of bundle of seven optical fibers. Six illumination fibers and one read fiber - each of which is $400 \mu \mathrm{m}$ in diameter. The fiber ends are coupled in such a manner that the 6-fiber leg (the illumination leg) is connected to the light source and the single fiber leg is connected to the spectrometer.

Acquisition of visible/near-IR data is straightforward. White light from a tungsten halogen lamp is brought to the skin via the reflectance probe. The light penetrates the skin, and water, hemoglobin species, cytochromes, lipids and proteins absorb this light at specific frequencies. The remaining light is scattered by the skin, with some light being scattered back to the fiber optic probe. The collector fiber in the reflectance probe collects the diffused light from the skin, and directs it to the spectrometer that generates the reflectance spectra with the spectral range of 400-1100 nm, which is then acquired on the computer. Prior to data acquisition, a reference spectrum is measured on a $\mathrm{BaSO}_{4}$ diffuse reflectance standard and all skin spectra were subsequently divided by this reference spectrum to ensure proper data calibration. This eliminates the experimental errors if any, present. Also, the instrument is subjected to gauge repeatability and reproducibility test, the results of which states that its reproducibility and repeatability is well with the acceptable range.

\section{Acquisition of spectra and analysis}

\subsection{Data acquisition}

Prior to obtaining the readings, the subject's skin and the end of the probe are cleansed with $70 \%$ alcohol. The fiber optic probe is then positioned $1.0 \mathrm{~mm}$ from the measurement site and data acquired. A plot of the amount of light backscattered at each wavelength (the spectrum) is computed. Measurements are rapid, non-destructive and non-invasive.

For the present study, different skin condition like - warts, vitiligo, thrombus (due to injury) and angioma are chosen. It is important to distinguish and diagnose various skin conditions. The diseased skin shows a very different molecular biology and biochemistry compared to normal skin.

Warts are small skin growths caused by viral infections. There over 100 types of human papilloma virus (HPV). Some warts share characteristics with other skin disorders such as molluscum contagiosum (a different type of viral skin infection), seborrhoeic keratosis (benign skin tumor) and squamous cell carcinoma (skin cancer). It is important to distinguish and diagnose.

Vitiligo is a zero melanin skin condition. The characteristics of this disease are the acquired sudden loss of the inherited skin color. The loss of the skin color yields white patches of various sizes, which can be localized anywhere on the body. However, not all white skin patches are vitiligo. There are other conditions and diseases that are associated with white skin called leucoderma. Clearly, it seems mandatory to make the correct diagnose.

Malignant Melanoma (MM) is another skin cancer which can be very dangerous if not recognized early. These tumors can develop in existing moles but they can also arise totally new as pigmented as well as non-pigmented tumors. Early recognition and excision are important for the outcome. The observation that melanoma is more frequent in patients with vitiligo originates from a study which included 623 Caucasian patients with melanoma of the Oncology Clinic at the Department of Dermatology at the University of Hamburg/Germany [22]. Some individuals with melanoma develop patches of white skin in the vicinity of their melanoma or after their tumor had been excised. In this context it seems 
important that these white patches are not vitiligo. This skin shows a very different molecular biology and biochemistry compared to true vitiligo [23].

Thrombus are the clumps that occur when the blood hardens from a liquid to a solid (coagulates). A thrombus, or blood clot, is the final product of the blood coagulation step in hemostasis. It is achieved via the aggregation of platelets that form a platelet plug, and the activation of the humoral coagulation system (i.e. clotting factors). A thrombus is normal in cases of injury, but pathologic in instances of thrombosis.

Angiomas are benign tumors that are made up of small blood vessels or lymph vessels. Cherry angiomas are made up of clusters of tiny capillaries at the surface of the skin, forming a small round dome ("papule"), which may be flat topped. They range in colour from bright red to purple.

Each reflectance spectrum is acquired by the diffuse reflectance spectroscopic system described in the previous section. Firstly, white light is directed into a portion of the skin afflicted with the skin disease, the diffusely reflected light is collected, thereby producing a condition spectrum. Next, the same light is directed into a control skin portion of the patient which is not afflicted with the skin disease. A spectrum is taken of an unaffected skin portion as a control from each patient.

\subsection{Data analysis}

The analysis is carried out for each diseased condition to obtain specific spectral signature by the following methods and the results are given in the next section.

(a) Ratio analysis technique. The ratio technique is used to aid spectra interpretation. In the ratio analysis technique, the lesional spectra are divided by the corresponding spectra of the normal neighboring skin. In this way, the relative spectral intensity changes are quantified and the spectral shape changes are enhanced and more easily visualized on the spectral curves. As a consequence, these differences can be used to identify or diagnose a skin disease by comparing the visible/near-IR spectrum of a control region to a spectrum taken on the region of interest.

(b) Student t-tests. Student's $t$-tests are performed on the obtained data to determine whether significant spectral differences existed between normal and diseased skin and whether spectra could be classified according to lesion type. The resulting $t$-values are plotted against wavelength. Student's $t$-tests showed significant differences $(p<0.05)$ between normal skin and varying skin conditions in several areas of the visible/near infrared spectrum.

(c) Difference spectra. Each mean condition spectrum is subtracted from each mean control spectrum in a pair-wise fashion to emphasize differences between spectra. This resulted in one difference spectrum for each case, representing spectral differences between the lesion and control site.

\section{Results and discussion}

As mentioned above, different skin abnormalities are studied viz. wart, angioma, thrombus and vitiligo regions. Figure 2 shows the plot of the original reflectance spectra of wart and the control skin along with the reflectance ratio between the wart region and the normal skin. The original spectra of wart show very low reflectance.

By obtaining the ratio spectrum we observe that there is a valley around $610 \mathrm{~nm}$ which is unique to wart and which cannot be discerned by only looking at the original spectrum. The values of the ratio plot are higher in the lower and higher wavelengths, except for the visible region. 


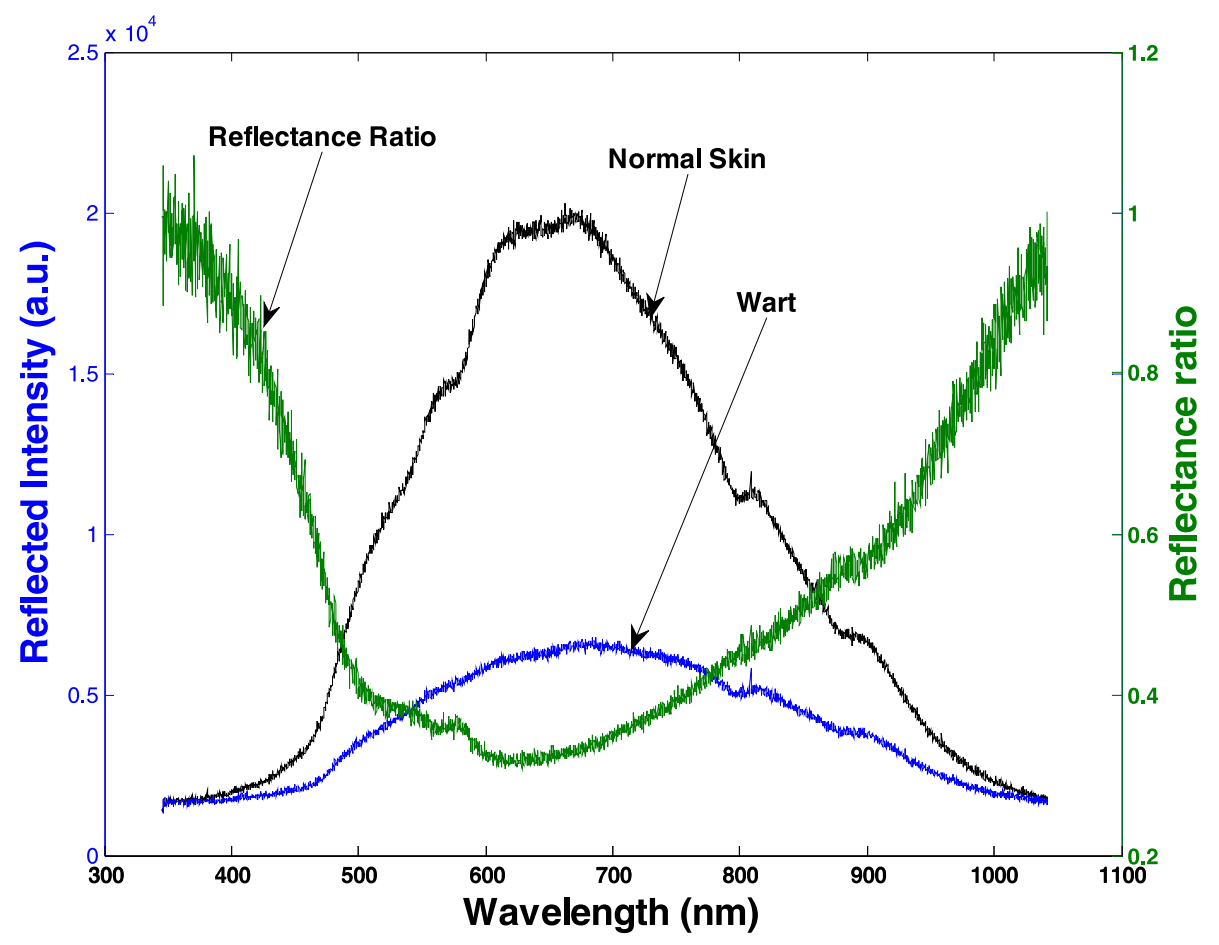

Fig. 2. Diffuse reflectance spectra for wart and the normal skin and their reflectance ratio spectra.

Figure 3 shows the reflectance spectra of vitiligo and the control skin along with their reflectance ratio. The absolute value of the ratio spectrum is larger than 1, indicating that the reflected intensity for a vitiligo skin is higher than that for the normal skin. Since vitiligo corresponds to region of zeromelanin skin, it has little absorption and hence maximum reflectance. The ratios with values less than 1 indicate that the lesional reflectance is lower than the surrounding normal skin. The numerical ratio values quantify this difference as a function of wavelength.

Figure 4 shows the reflectance spectra of thrombus and the control skin along with their reflectance ratio. Looking at the spectra we observe that the shapes of the two curves are visually same except for small changes in reflected intensity values. We can see valleys around the two secondary hemoglobin absorption bands at 542 and $577 \mathrm{~nm}$. As thrombus is the clumps that occur when the blood hardens the absorption of blood plays a role in depicting the shape of the ratio spectrum.

Figure 5 shows the reflectance spectra of angioma and the control skin along with the reflectance ratio. To quantify the spectra, ratio analysis is carried out. It shows that the specific wavelength is around 590 $\mathrm{nm}$, which is again one of the important absorption band of hemoglobin.

Student $t$-test is also performed on those spectra for further analysis. Figure 6 shows the plot of the mean original reflectance spectra of wart and the control skin along with the $t$-value plot. The resulting $t$-values are plotted against wavelength. Student's $t$-tests showed significant differences $(p<0.05)$ between normal skin and wart region in several areas of the visible/near infrared spectrum. As seen in the reflectance plot the same wavelength of $610 \mathrm{~nm}$ seems to be the specific wavelength for the wart under study.

Figure 7 shows the plot of the mean original reflectance spectra of vitiligo and the control skin along with the $t$-values. From the $t$-value plot we observe that visible red region and near IR wavelengths 


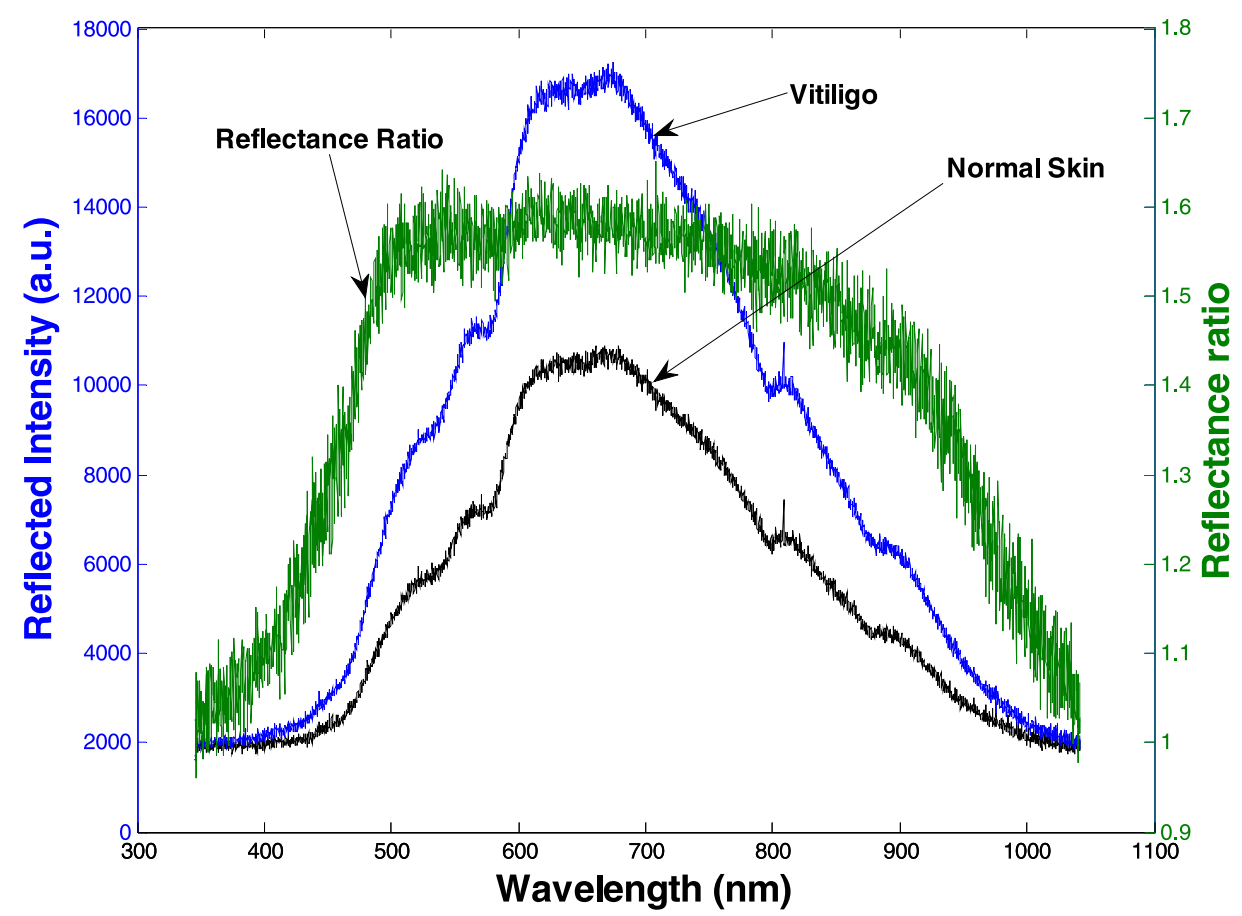

Fig. 3. Diffuse reflectance spectra for vitiligo and the normal skin and their reflectance ratio spectra.

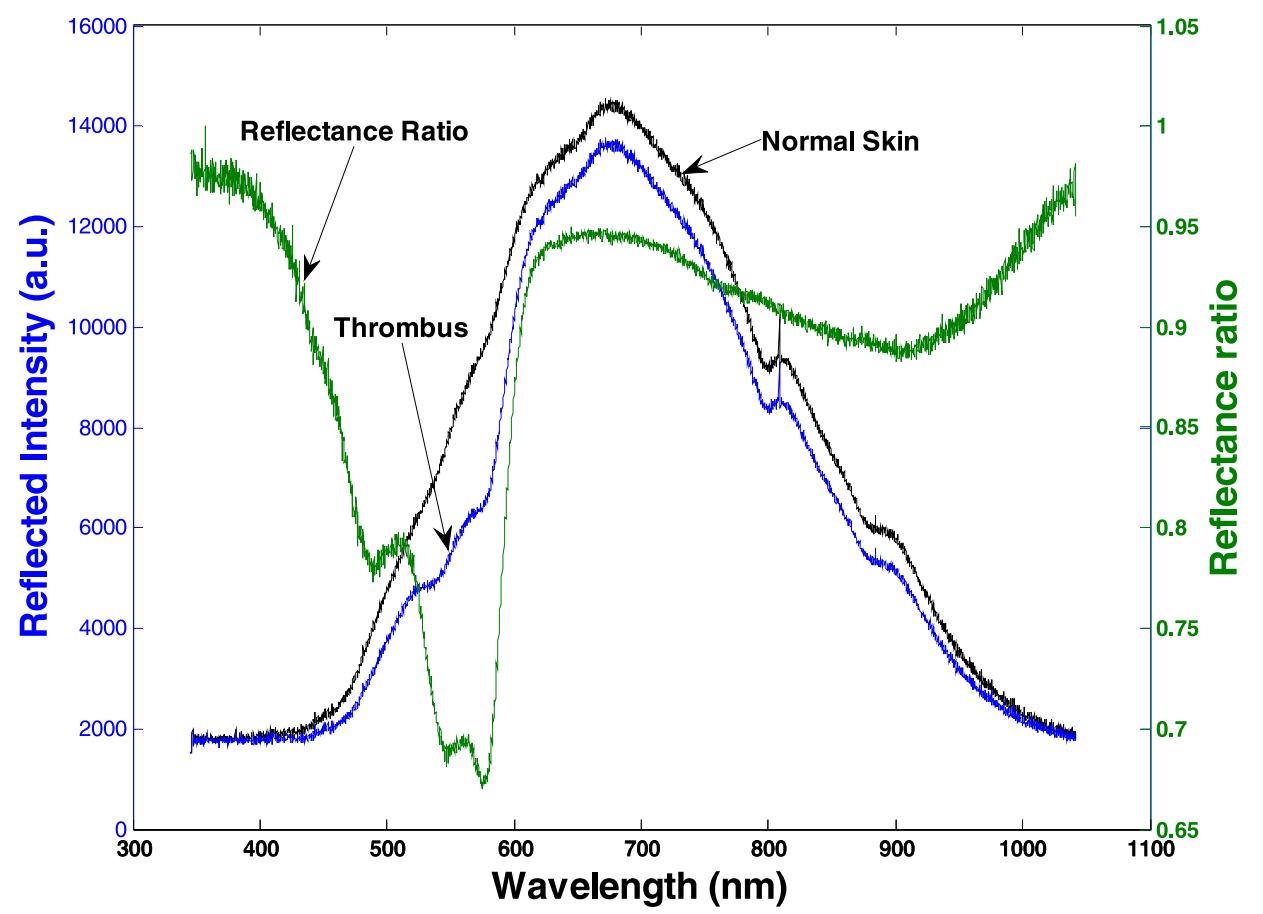

Fig. 4. Diffuse reflectance spectra for thrombus and the normal skin and their reflectance ratio spectra. 


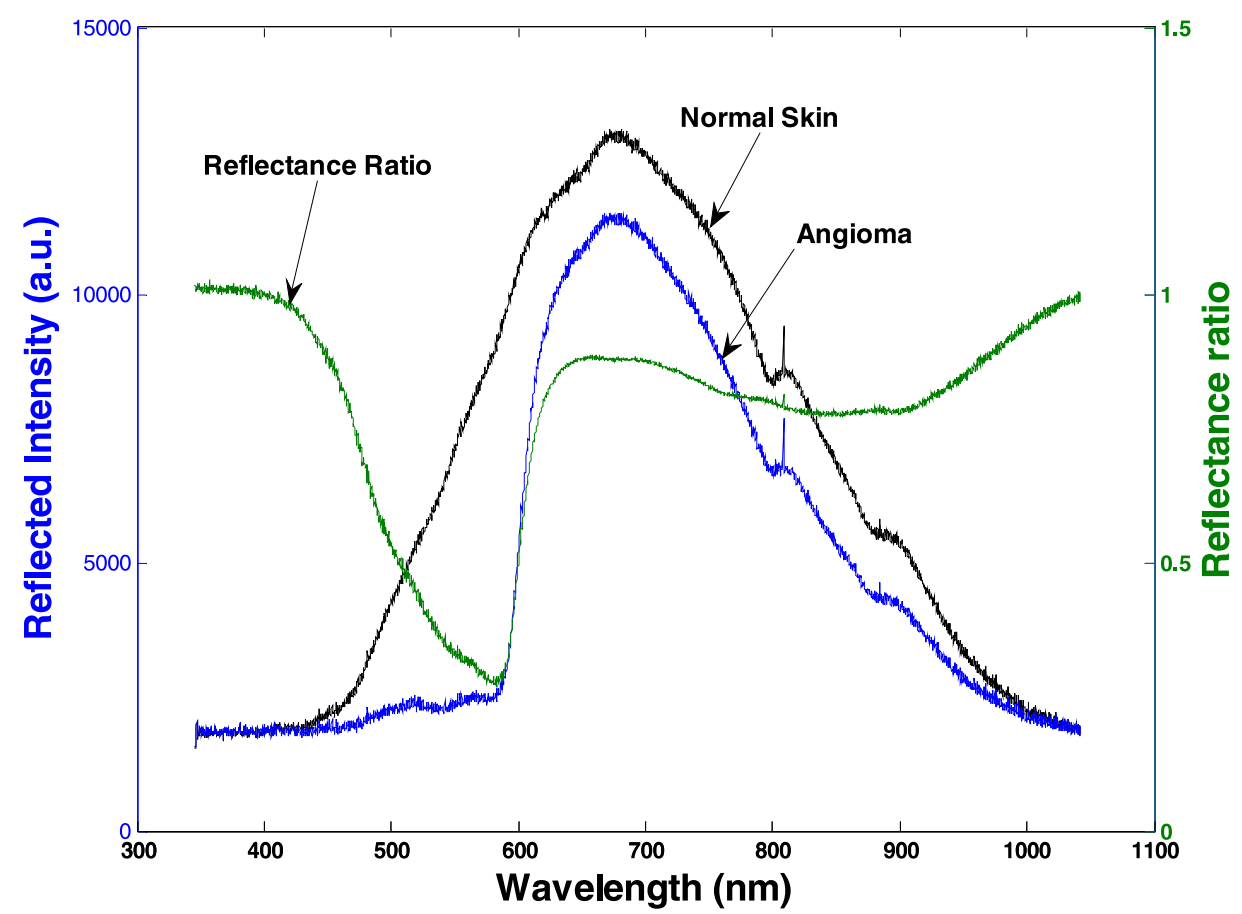

Fig. 5. Diffuse reflectance spectra for angioma and the normal skin and their reflectance ratio spectra.

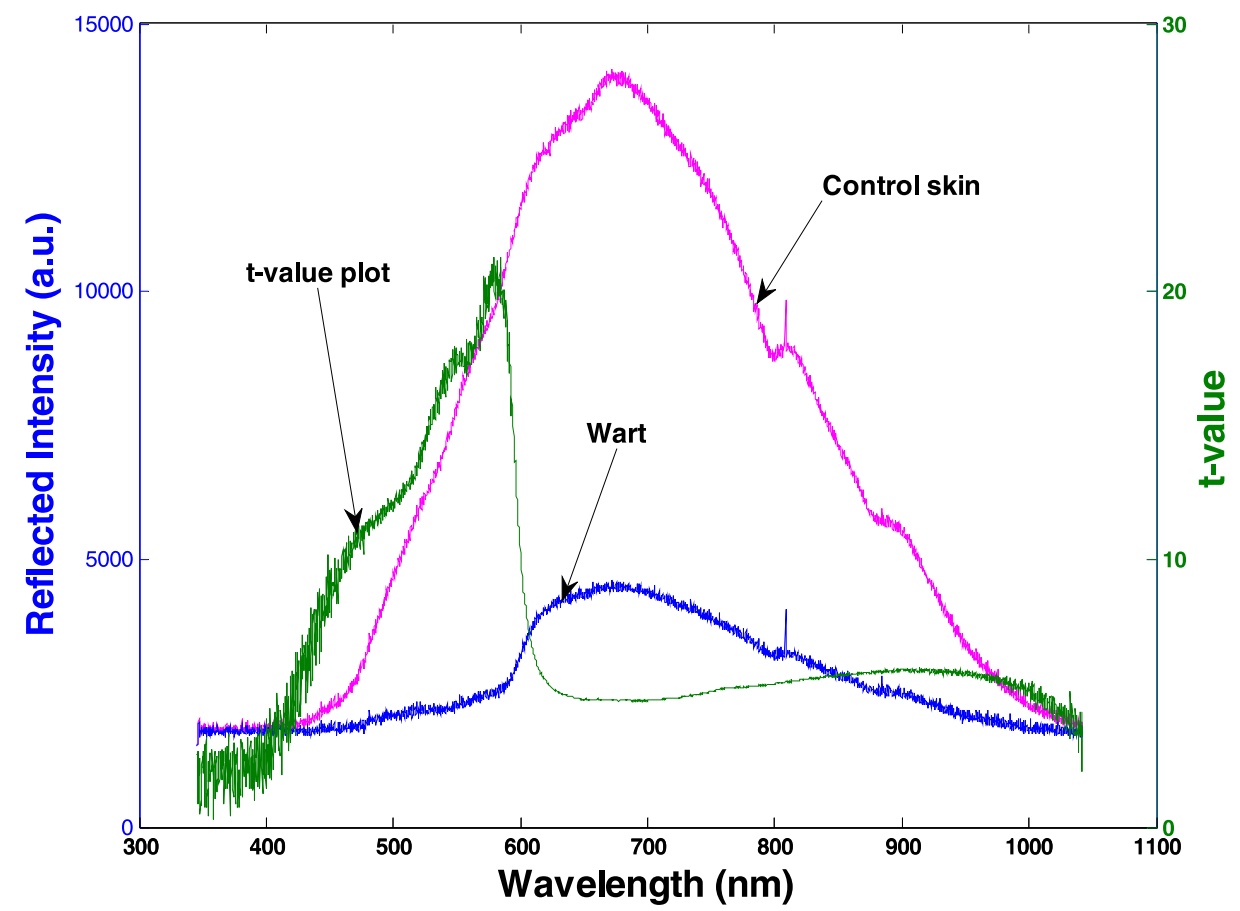

Fig. 6. The mean control spectra and condition spectra for wart shown overlaid on $t$-value traces. 


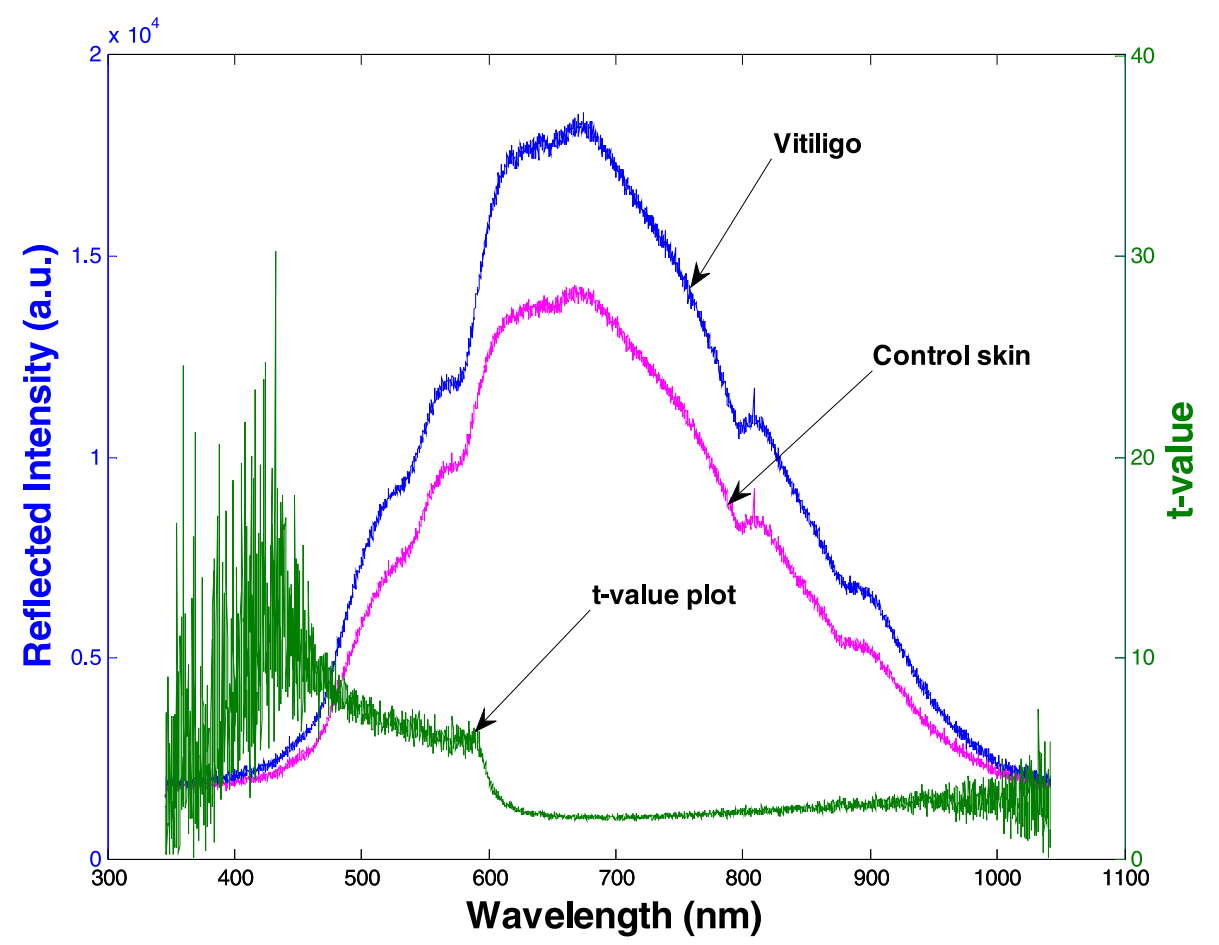

Fig. 7. The mean control spectra and condition spectra for vitiligo shown overlaid on $t$-value traces.

are the important region. As the chromophores melanin has characteristic absorption in this wavelength region.

Figure 8 shows the plot of the mean original reflectance spectra of thrombus and the control skin along with the $t$-values. The thrombus region shows little variation in reflectance values but the shapes of the two curves are visually same. The relative spectral intensity changes are quantified and the spectral shape changes are enhanced and more easily visualized on the spectral curves. We observe that 540 $580 \mathrm{~nm}$ range of wavelength is of importance. It is interesting to note that the extinction coefficient of de-oxyhemoglobin is higher in this range of wavelength as well.

Figure 9 shows the plot of the original reflectance spectra of angioma and the control skin along with the $t$-values. As such there is not much difference in the shapes of the two spectral curves, but the reflected intensity of the angioma is found to be low. The $t$-value plot beyond $580 \mathrm{~nm}$ matches with the oxyhemoglobin's extinction spectra till around $640 \mathrm{~nm}$. Further analyses are to be carried out before we can identify any region of significance.

As mentioned in the previous section, four different skin conditions are studied. Apart from the ratio analysis and Student's $t$-tests, difference plot is made for those skin conditions under study. Diffuse reflectance spectra of various skin conditions showed consistent differences from that of normal tissue. Reflectance intensity of abnormal tissue was lower than that of normal tissue. The control spectra and the disease spectra in each skin condition group in the database are then reduced to diagnostic wavelengths. As a consequence, on further analysis, these differences can be used to identify or diagnose a skin condition by comparing the visible/near-IR spectrum of a control region to a spectrum taken on the region of interest. 


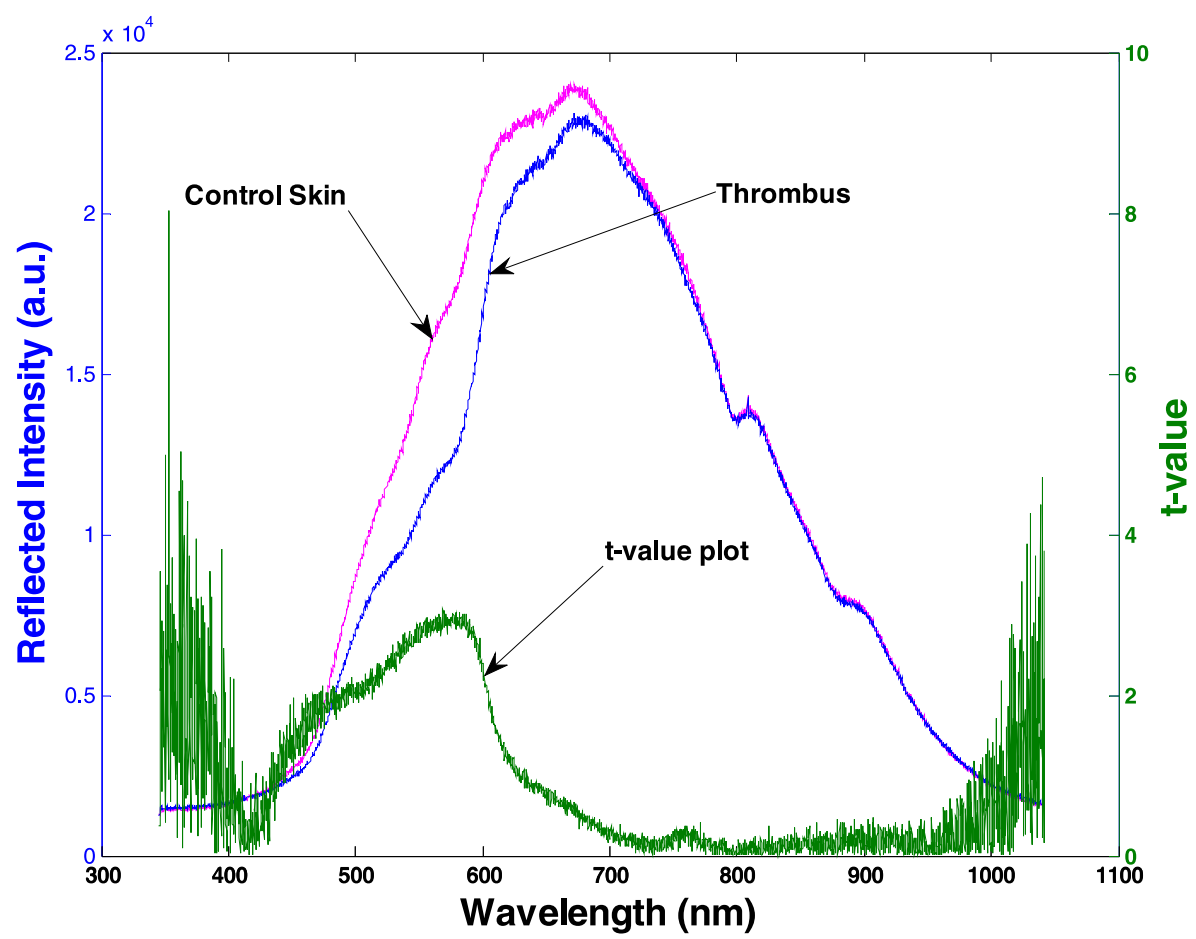

Fig. 8. The mean control spectra and condition spectra for thrombus shown overlaid on $t$-value traces.

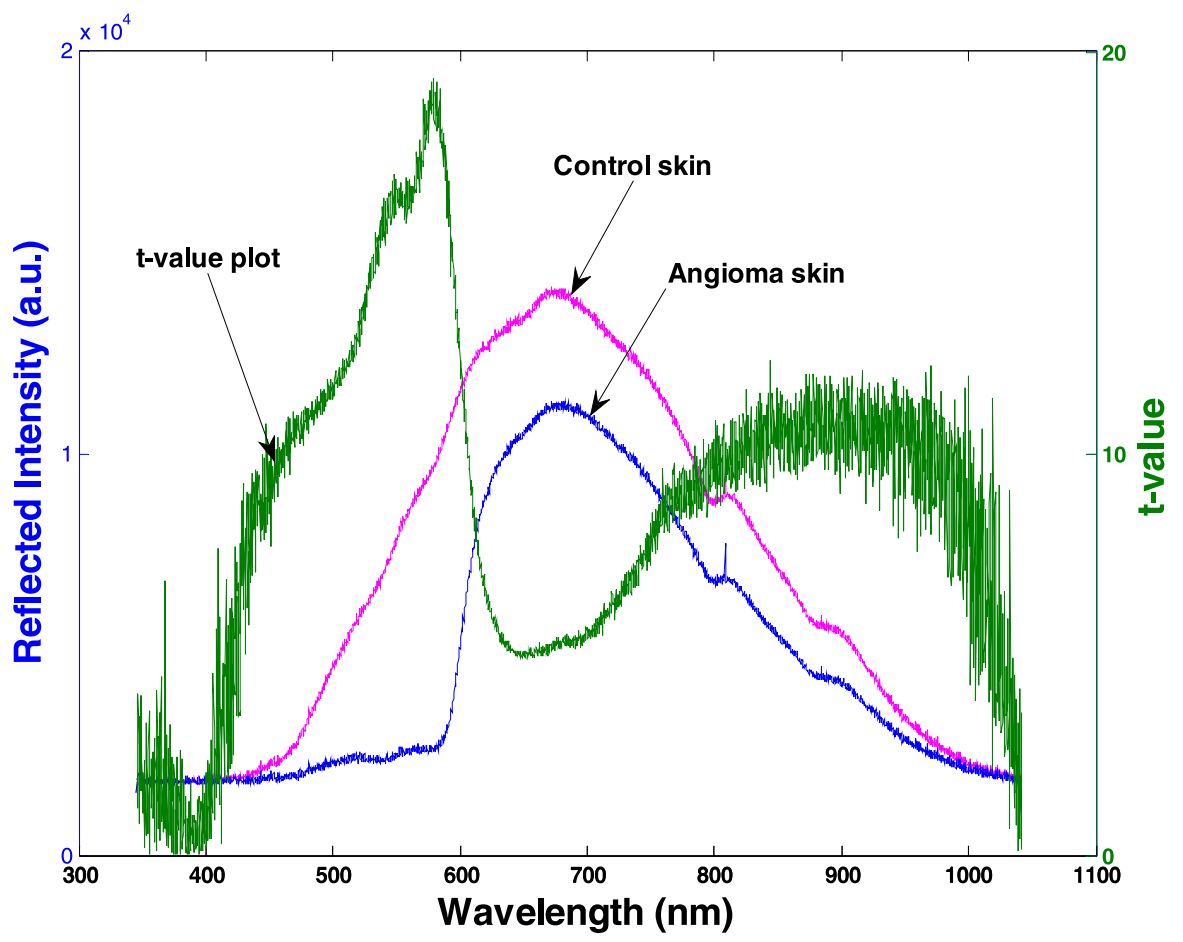

Fig. 9. The mean control spectra and condition spectra for angioma shown overlaid on $t$-value traces. 


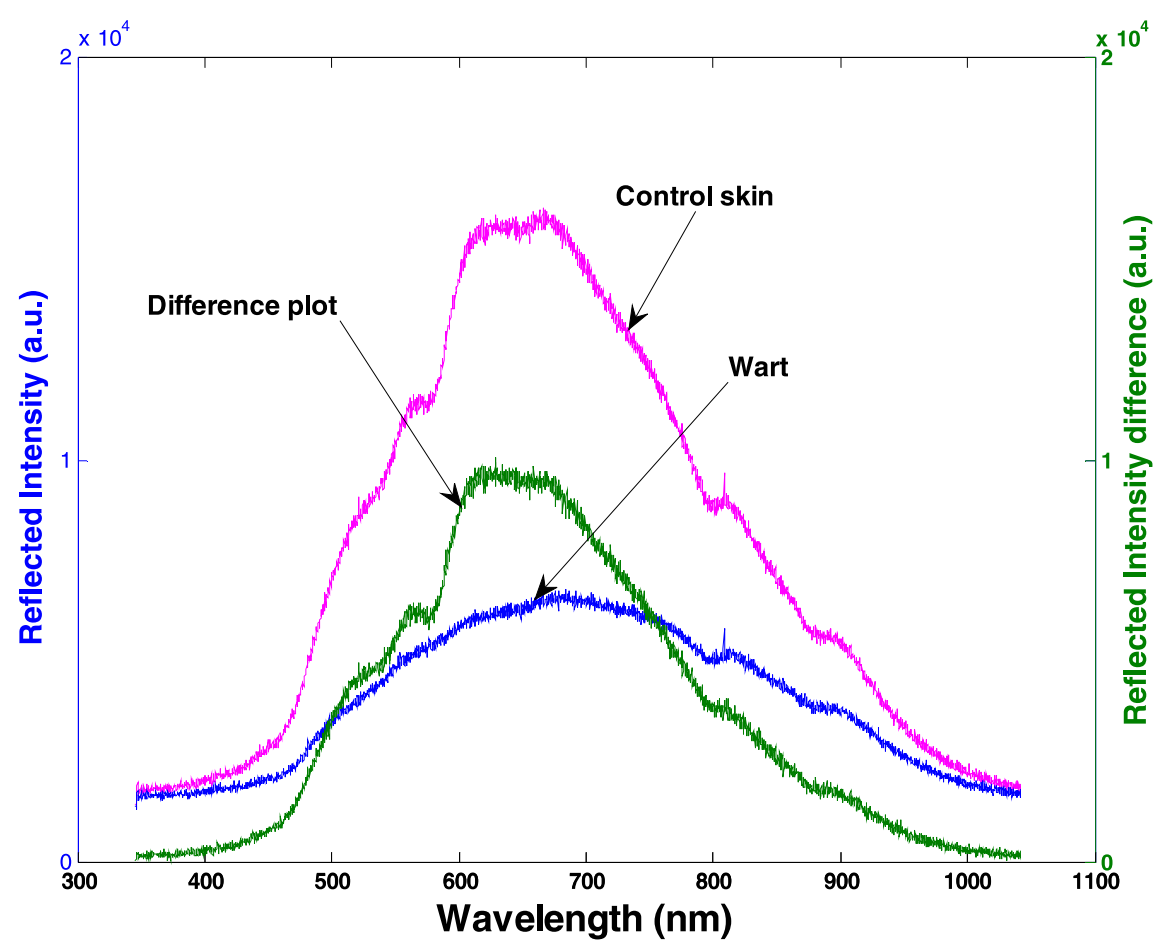

Fig. 10. The plot of the mean original reflectance spectra of wart and the normal skin along with their difference plots.

Figure 10 shows the plot of the mean of original reflectance spectra of wart and the normal skin along with their difference plots. Difference plot like the ratio plot and $t$-value plot shows the peak wavelength is $610 \mathrm{~nm}$ for this wart under study.

Figure 11 shows the plot of the mean of original reflectance spectra of vitiligo and the normal skin along with their difference plots. Since the reflectance for the vitiligo skin is higher than the normal skin the difference plot is negative. Here again the maximum difference is found around the red-near-IR region of spectrum.

Figure 12 shows the plot of the mean of original reflectance spectra of thrombus and the normal skin along with their difference plots. From the mean curves we observe that beyond $700 \mathrm{~nm}$ both the curves more or less overlap. From the difference spectrum we observe that $540-585 \mathrm{~nm}$ range of wavelength is of importance, the $t$-value plot also reflects the same. Changes in hemoglobin content in dermis lead to changes in absorption in these wavelength regions.

Figure 13 shows the plot of the mean of original reflectance spectra of angioma and the normal skin along with their difference plots. As can be seen throughout the spectrum the control spectral intensity is higher. In the wavelength region $420-580 \mathrm{~nm}$ there is a flat response in the original spectrum but a sharp increase in the difference spectrum. As can be seen the difference plot shows much variation in the reflectance values in the wavelength region of $480-580 \mathrm{~nm}$. Since angioma is full of blood capillaries, the absorption by hemoglobin results in the variation in the reflectance values in these regions. The wavelength region of $650-700 \mathrm{~nm}$ can be studied to estimate the melanin index. As vitiligo is a region of zero melanin compared with that of a normal skin, the slope of these regions gives an estimation of the melanin index. The hemoglobin absorption is studied by analyzing the $535-585 \mathrm{~nm}$ region. 


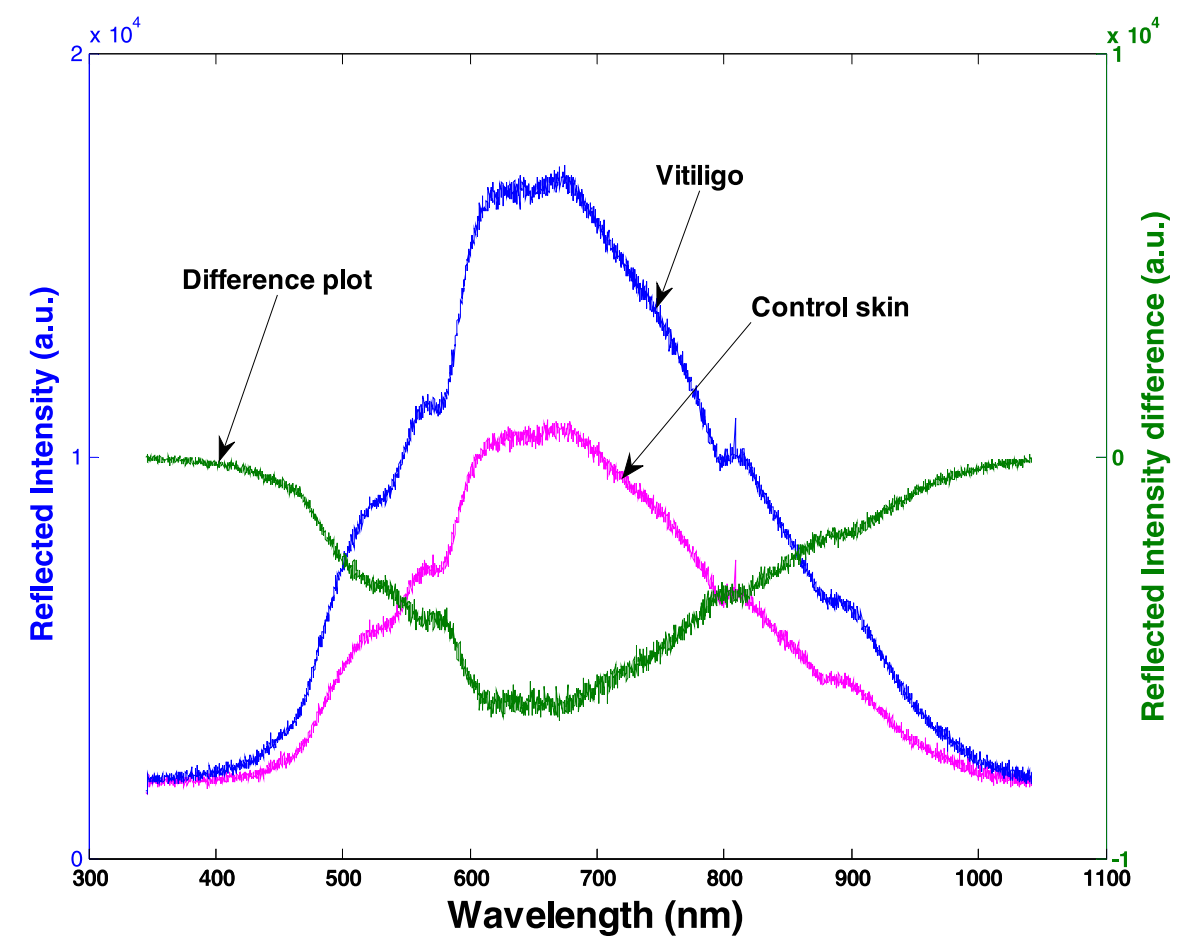

Fig. 11. The plot of the mean original reflectance spectra of vitiligo and the normal skin along with their difference plots.

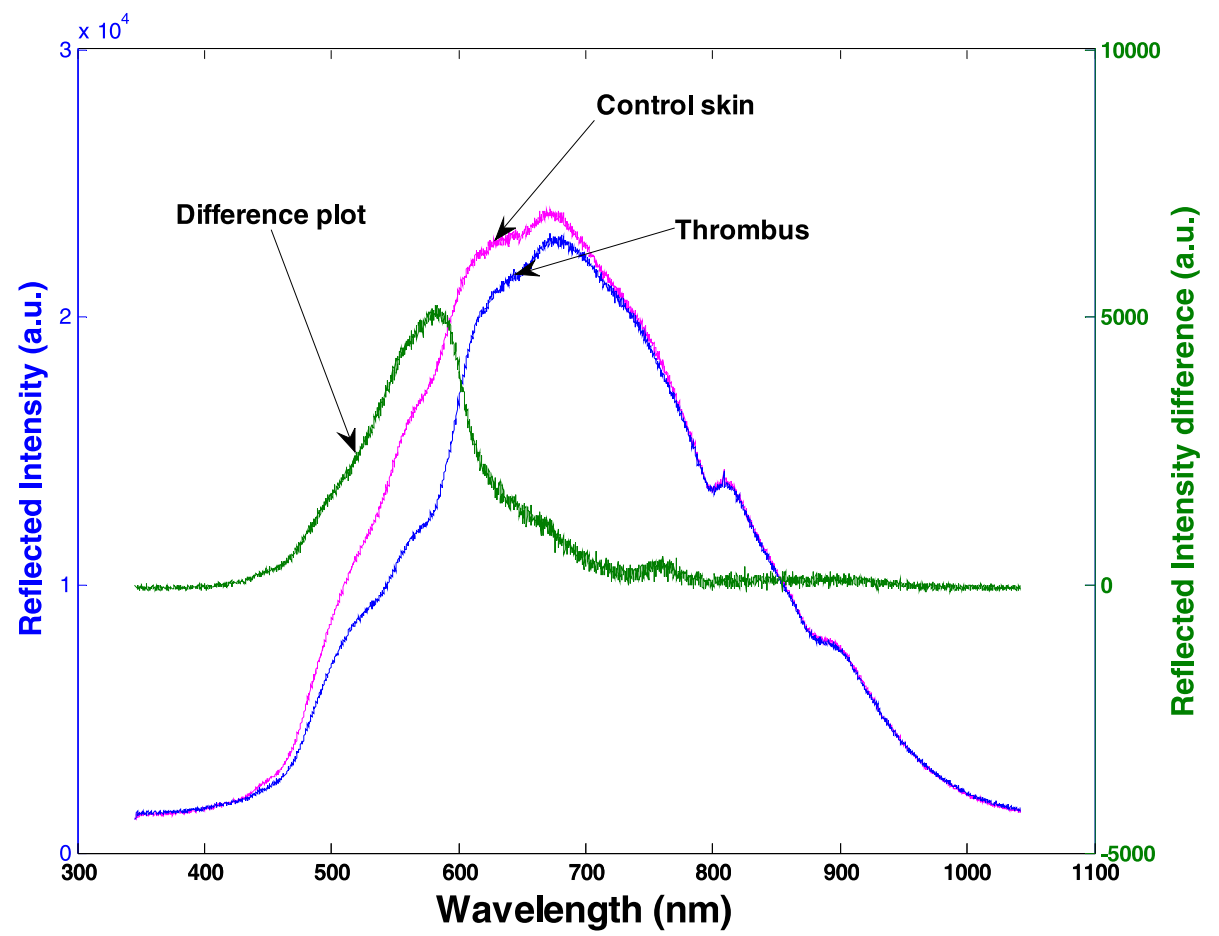

Fig. 12. The plot of the mean original reflectance spectra of thrombus and the normal skin along with their difference plots. 


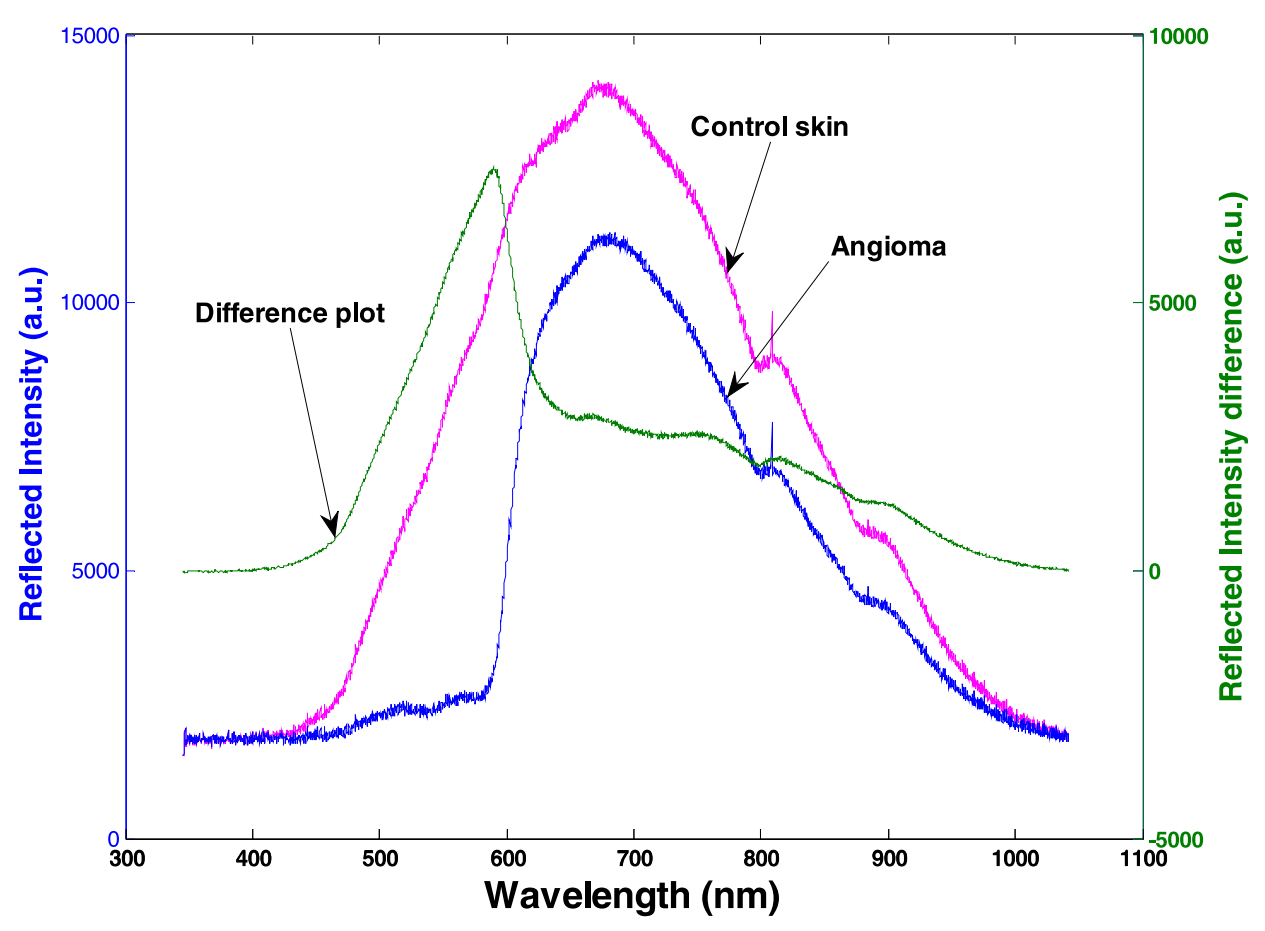

Fig. 13. The plot of the mean original reflectance spectra of angioma and the normal skin along with their difference plots.

As can been seen from the above results, spectral analysis of the reflectance data is important to characterize a tissue. As, the optical properties like absorption coefficient $\left(\mu_{\mathrm{a}}\right)$ and scattering coefficient $\left(\mu_{\mathrm{s}}\right)$ are wavelength dependent, if a specific wavelength region is identified for a disease through the above techniques then, their optical properties can be compared at those wavelengths for normal and diseased tissues for diagnostic applications. Also identifying significant regions of interest and their study will help us to differentiate various skin conditions and thereby aid in better diagnosis.

The visible/near-IR spectra of different skins presented here exhibit a number of weak, but consistent, absorption bands arising from oxy- and deoxy- hemoglobin, lipids and proteins. However, visual examination of spectra did not show distinct differences in these spectral features that could be used to distinguish between spectra of skin diseases and healthy skin.

\section{Conclusions}

The spectrum depends on the depth and the type of chromophore contained in the inclusion. An increase in the concentration of a given molecule may produce different contrast, independently of the depth, depending on the characteristics of the skin layer where this change occurs. For the type of wart under study the identified wavelength is $610 \mathrm{~nm}$. For the thrombus tissue the spectral region of interest is $540-585 \mathrm{~nm}$, and angioma $580-590 \mathrm{~nm}$.

The disease spectra and the control spectra are compared using statistical analysis to detect wavelength regions of significant difference between the control spectra and the lesion spectra. The difference plot between diseased spectra and the normal spectra help us to detect wavelength regions of significant difference between the normal spectra and the diseased spectra. 
Previously, we have presented preliminary studies that assess the diagnostic power of diffuse reflectance spectroscopy. Visible/near-infrared spectroscopy is a promising non-invasive technique for the screening of skin diseases.

Persistent spectral patterns demonstrated that the contribution of hemoglobin absorption and the wavelength-dependent spectral slope will contain relevant information for classification. For instance, the relatively strong absorption feature between $535-585 \mathrm{~nm}$ arises from hemoglobin species and provides information relating to the oxygenation status of tissues. Changes in tissue architecture/optical properties may affect the basic nature of the interaction of light with the tissue. For example changes in the character of the epidermis (i.e. dehydration) may result in more scattering of light from the surface, reducing penetration of light into the skin in a wavelength-dependent manner. Such phenomena would be manifest in spectra as changes in the slope of the spectral curves, especially in the 400-780 nm regions.

Future study includes the extraction of functional parameters like oxygen saturation, melanin content and oxygen concentration along with the optical properties at specific wavelengths for each type of tissue under study. This method can be extended to cancerous tissues as well and diagnose it prematurely enabling better treatment and minimize patient trauma.

\section{Acknowledgement}

This research work is being funded and supported by All India Council for Technical Education (AICTE), Government of India, New Delhi under the scheme of Career Award for Young Teacher (CAYT).

\section{References}

[1] N. Shah, A.E. Cerussi, D. Jakubowski, D. Hsiang, J. Butler and B.J. Tromberg, The role of diffuse optical spectroscopy in the clinical management of breast cancer, Disease Markers 19 (2003/04), 95-105.

[2] S.C. Bruce et al., Functional near Infrared spectroscopy, IEEE Eng. in Med. and Biol. Magazine 25(4) (2006), 54-62.

[3] R. Manoharan, K. Shafer, L. Perelman, J. Wu, K. Chem, G. Deinum, M. Fitzmaurice, J. Myles, J. Crowe, R.R. Dasari and M.S. Feld, Raman spectroscopy and fluorescence photon migration for breast cancer diagnosis and imaging, Photochem. Photobiol. 67 (1998), 15-22.

[4] N.T. Yu, B.S. Krantz, J.A. Eppstein et al., Development of noninvasive diabetes screening device using the ratio of florescence to Rayleigh scattered light, J. Biomed. Opt. 11 (1996), 243-250.

[5] N.B. Colthup, L.H. Daly and S.E. Wiberley, Introduction to Infrared and Raman Spectroscopy, 3rd edn, Academic Press, New York, 1990.

[6] T. Vo-Dinh, Biomedical Photonics Handbook, CRC Press, 2003.

[7] G. Zonios, L.T. Perelman, V. Backman et al., Diffuse reflectance spectroscopy of human ademonatous colon polyps in vivo, Appl. Opt. 38 (1999), 6628.

[8] J.R. Mourant, I. Bigio et al., Spectroscopic diagnosis of bladder cancer using elastic scattering spectroscopy, Lasers Surg. Med. 17 (1995), 350.

[9] I. Bigio et al., Diagnosis of breast cancer using elastic scattering spectroscopy: preliminary clinical results, J. Biomed. Opt. 5 (2000), 221.

[10] V. Wallace et al., Classification of reflectance spectra from pigmented skin lesions, a comparison of multivariate discriminant analysis and artificial neural networks, Phys. Med. Biol. 45 (2000), 2859.

[11] S. Prince and S. Malarvizhi, Monte Carlo simulation of NIR diffuse reflectance in the normal and diseased human breast tissues, Biofactors 30 (2007), 255-263.

[12] P.J. Dwyer, R.R. Anderson and C.A. DiMarzio, Mapping blood oxygen saturation using a multi-spectral imaging system, in: Biomedical Sensing, Imaging, and Tracking Technologies II, T. Vo-Dinh, et al., eds, Proc. Soc. Photo-Opt. Instrum. Eng., vol. 2976, SPIE, International, Bellingham, WA, 1997, pp. 270-280. 
[13] S. Prince and S. Malarvizhi, Functional optical imaging of a tissue based on diffuse reflectance with fibre spectrometer, in: 4th European Congress for Medical and Biomedical Engineering, IFMBE Proceedings, Vol. 22, Springer, BerlinHeidelberg, 2008, pp. 484-487.

[14] S. Prince and S. Malarvizhi, Multi wavelength diffuse reflectance plots for mapping various chromophores in human skin for non-invasive diagnosis, in: The 13th International Conference on Biomedical Engineering 2008, IFMBE Proceedings, Vol. 23, Springer, Berlin-Heidelberg, 2009, pp. 323-326.

[15] R.R. Anderson and J.A. Parrish, Optical properties of human skin, in: The Science of Photomedicine, J.D. Regan and J.A. Parrish, eds, Plenum Press, New York, 1982, pp. 147-194.

[16] R. Marchesini, C. Clemente, E. Pignoli and M. Brambilla, Optical properties of in vivo epidermis and their possible relationship with optical properties of in vivo skin, Photochem. Photobiol. 16 (1992), 127-140.

[17] M.J.C. van Gemert, S.L. Jacques, H.J.C.M. Sterenborg and W.M. Star, Skin optics, IEEE Trans. Biomed. Eng. 36(12) (1989), 1146-1154.

[18] S. Prince and S. Malarvizhi, Estimation of chromophores through skin modeling and Monte Carlo simulation of its spectral characteristics, International Journal of Computational Intelligence and Healthcare Informatics 1 (2008), 13-17.

[19] A. Welch and M. van Gemert, Optical-Thermal Response of Laser-Irradiated Tissue - Lasers, Photonics and Electrooptics, Plenum Press, 1995.

[20] V.V. Tuchin, Lasers and Fiber Optics in Biomedical Science, Saratov Univ. Press, Saratov, 1998.

[21] Ocean Optics, http://www.oceanoptics.com/products.

[22] K.U. Schallreuter, C. Levenig and J. Berger, Vitiligo and cutaneous melanoma, Dermatologica 183 (1991), $239-245$.

[23] S. Hasse, S. Kothari, H. Rokos, S. Kauser, N.Y. Schurer and K. Schallreuter, In vivo and in vitro evidence for autocrine DCoH/HNF-1 [alpha] transcription of albumin in the human epidermis, Experimental Dermatology 14(3) (2005), 182-187. 


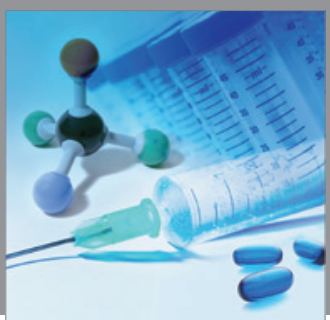

International Journal of

Medicinal Chemistry

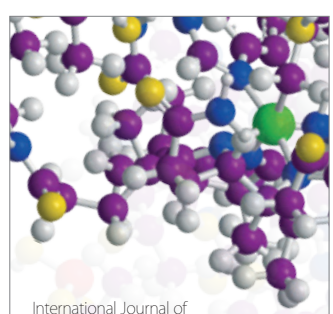

Carbohydrate Chemistry

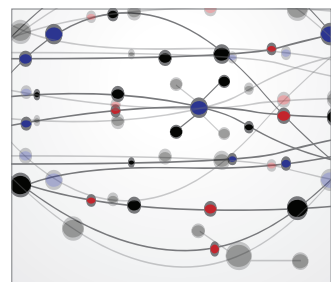

The Scientific World Journal
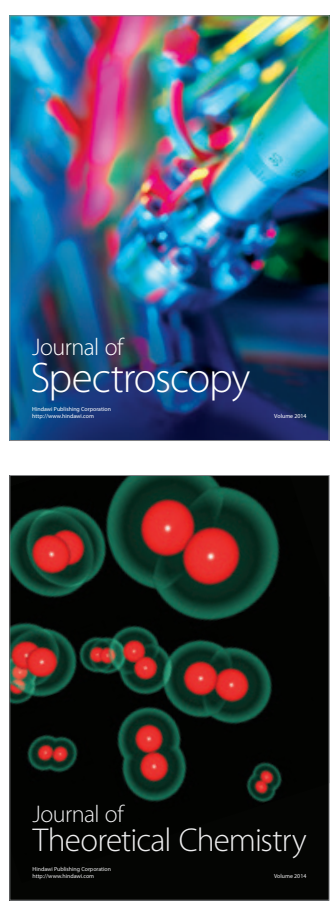
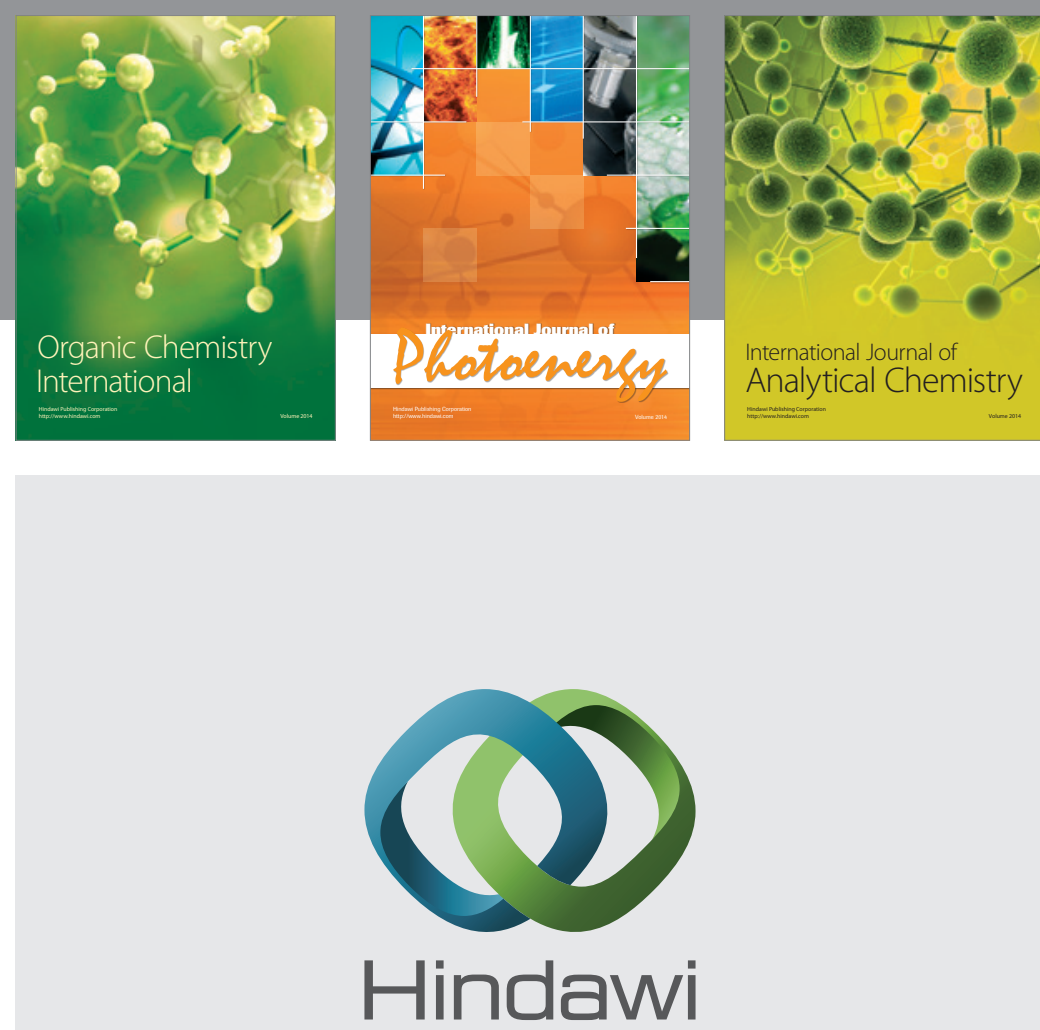

Submit your manuscripts at

http://www.hindawi.com
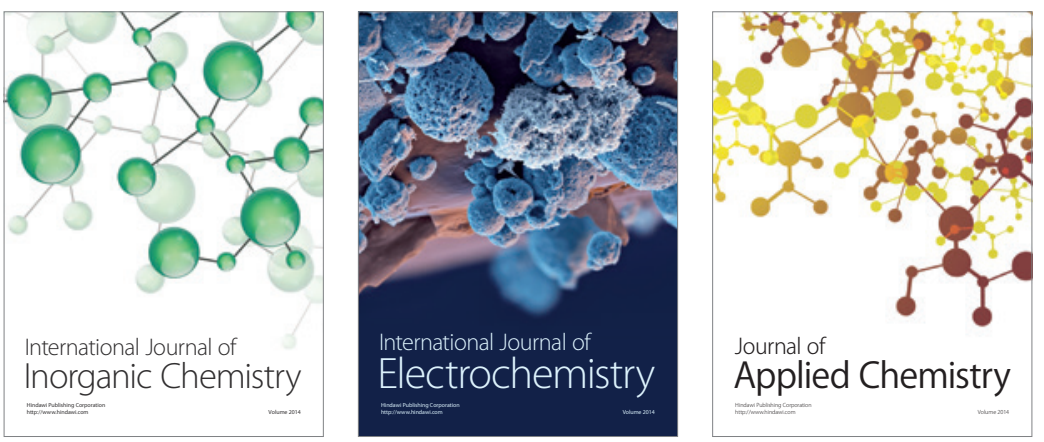

Journal of

Applied Chemistry
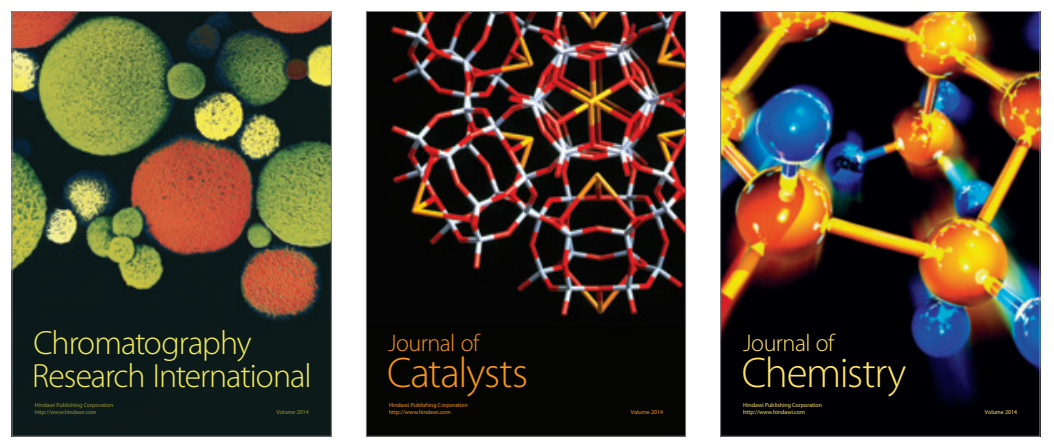
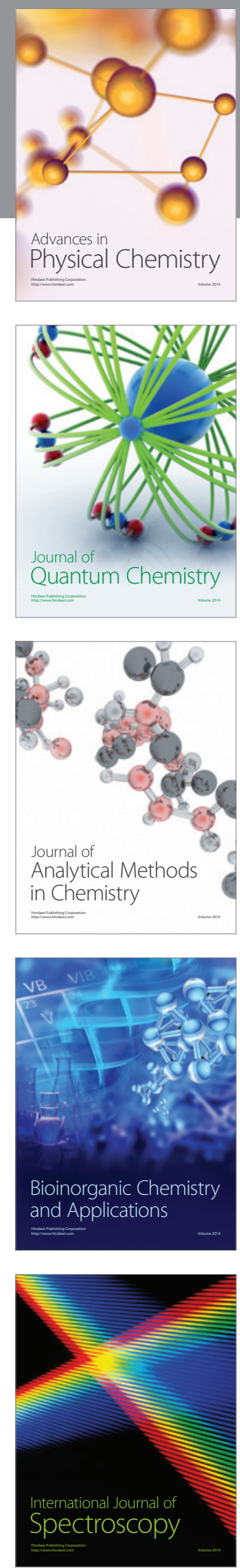